\title{
Design and Simulation of PEM Control System for Reactive Sputtering Coating
}

\author{
Ying Huang ${ }^{1, a}$, Jinyan Zhao ${ }^{1, b}$, Caihong Chen ${ }^{1, \mathrm{c}}$ and Yichen Zhang ${ }^{1, \mathrm{~d}}$ \\ ${ }^{1}$ School of Mechanical Engineering \& Automation, Northeastern University, Shenyang 110819, PR \\ China \\ ayinghuang@me.neu.edu.cn, 'beuzjy@126.com, 'chencaihong3@sina.com, \\ dychzhang@me.neu.edu.cn
}

Keywords: Reactive sputtering, PEM, Single neuron adaptive PID, plasma, OES

\begin{abstract}
During the reactive sputtering process, due to the hysteresis effect, the sputtering state should be maintained in the transition region of the hysteresis curve which can used to obtain stoichiometric compound films at a high deposition rate. If sputtering state changes, it is impossible to make the sputtering state step back to the original point by manually control the process parameters, because the hysteresis is irreversible. Thus it requires a method of fast feedback to control the sputtering power and the reaction gas flow rate into the chamber. In this paper the PEM (plasma emission monitor) control system and the single neuron self-adaptive PID algorithm have been designed to maintain the sputtering state in proper condition, namely preventing the target from poisoned in the reactive sputtering. The signal acquisition and the controller design were the major parts of the PEM system. The signal acquisition was realized by the optical emission spectrometer. And the single neuron self-adaptive PID controller has been designed in the paper. Using the MATLAB software, the simulation experiments have been done. The output waveforms showed that using traditional non-adaptive PID control algorithm, the overshoot is over $6 \%$ and the regulation time is over $1.8 \mathrm{~s}$, but using single neuron self-adaptive PID algorithm the overshoot 0 and regulation time $0.5 \mathrm{~s}$. Monitoring the target spectral intensity at various reaction gas flow rate, several conclusions could be obtained. The overshoot $6 \%$ indicated that the reactive gas flow into the chamber was excessive, the target was poisoned and the sputtering state in chemical mode. And while the overshoot was zero which indicated that the target poisoned was avoided and the reaction ran in defined condition. The PEM using the single neuron self-adaptive PID algorithm responded faster than that using the traditional PID algorithm. The PEM system designed in the paper can effectively avoid the target poisoned and make the reactive sputtering maintain at an ideal state.
\end{abstract}

\section{Introduction}

Reactive sputtering is one of the most commonly used techniques for obtaining compound thin films including oxides, nitrides, carbides or arsenides by sputtering metal targets in reactive gases. The reaction not only takes place on the substrate but also on the target. It is the reaction on the target surface that leads to the main reactive sputtering problems, such as target poisoning, arc and hysteresis effect. It is possible to sputter compound materials from a poisoned target, but the deposition rate is usually very low. The ideal sputtering processes is in the transition region between the elemental and poisoned states of the target to gain a higher deposition rate and optimum film properties, but it is very unstable so that fast process control systems are required in order to operate inside this region. Until now plasma emission monitoring (PEM) and voltage control techniques 
have proved to be very reliable in controlling reactive sputtering processes. PEM shows more obvious advantages over voltage control because the difference between emitted intensity of the metallic target and that of the reaction product is sufficiently large and positive.

In this paper, a PEM control system and proper algorithm are designed to control the reactive gas flow rate for the purpose of maintaining the sputtering state in the transition region to prevent the target from poisoning in the reactive sputtering.

\section{PEM system design and simulation principles}

Choice of set-point of the PEM. The Set-point (SP) is the most critical parameter for the PEM control system which directly determines the reliability, sputtering rate and properties of film prepared. In order to get the standard stoichiometric compound films, the SP should be within a certain range. It only makes sense to increase the deposition rate on the premise that correct stoichiometric can be obtained.

Although it is possible in principle for PEM system to monitor different emission lines as long as they change with the sputtering state, target atomic emission lines are mostly chosen as control signals for the intensities of these lines are intensive and remarkable. They are not usable for process control if the emission lines show very low intensities. The ratio of actual intensity to intensity of pure metallic sputtering is mostly used as the set-point, which indicates the degree of reaction at the cathode target surface.

System design and operation principle. Referring to the operational principles of the PEM and literatures[2-7], the whole system is designed as the design block diagram showed in Fig. 1. AvaSpec-2048FT optical spectrometer and the matching software AvaSoft-Basic are used to measure the emission spectra. Certain intensity of spectral line can be extracted or furthermore be calculated as the regulating parameter.

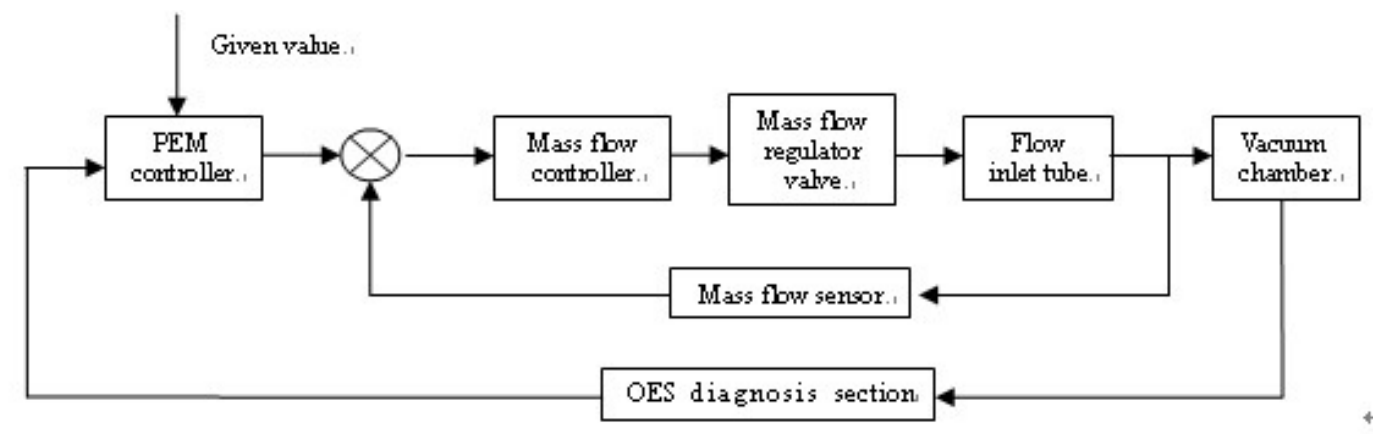

Fig. 1. System design block diagram

The system works as follows. The optical fiber probe is fixed in front of the observation window, aiming at the bright negative glow discharge area to collect the plasma emission light signals. The light signals are transmitted through the optical fiber into the spectrometer in which the light signals are diffracted and interfered into line spectra of different wavelengths. Then the line spectra are transformed into through photoelectric tube or CCD components electrical signals which are compared with the given value and error signals are figured out by the PEM controller. At last error signals control the flow rate of the reactive gas to increase or decrease into the vacuum chamber. Thereby the sputtering states maintain in the transition region while the deposition rate and film stoichiometry stay at proper value. 
The principle of algorithm simulation. The conventional PID continuous formula is given by:

$$
u(t)=K_{p}\left[e(t)+\frac{1}{T_{i}} \int_{0}^{t} e(t) d t+T_{d} \frac{d e(t)}{d t}\right]
$$

where, $K_{p}$ : the proportional gain, $T i$ : integral gain, $T d$ : derivative gain.

When a shorter sampling period To is chosen, the discrete formula is:

$$
u(t)=K_{i} e(k)+K_{p} \Delta e(k)+K_{d} \Delta^{2} e(k)
$$

The structure chat of the adaptive PID control by single neuron is shown as Fig. 2.

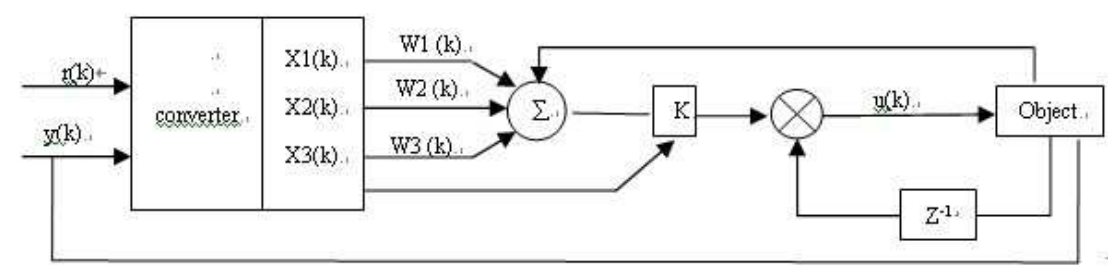

Fig. 2. A single neuron adaptive PID controller block diagram

The inputs of the converter shown in Fig. 2 are set-point $r(k)$ and output $y(k)$, while the outputs are $x 1, x 2$ and $x 3$ separately standing for quantities of state needed by neuron study control, also inputs of single neuron. The control signal, $\mathrm{u}(\mathrm{k})$, is generated by neuron using the methods of associate search and self-study.

Here

$x_{1}(k)=r(k)-y(k)=e(k) \quad, \quad x_{2}(k)=e(k)-e(k-1) \quad, \quad x_{3}(k)=e(k)-2 e(k-1)+e(k-2) \quad$, $Z(k)=r(k)-y(k)=e(k)$.

$\mathrm{Z}(\mathrm{k})$ is performance index or hierarchical signal, $k$ is proportionality coefficient of neuron, $k>0$. In Matlab, the track to realize state converting with zero-order holder is: input $\mathrm{e}(\mathrm{k})$, save its previous state $\mathrm{e}(\mathrm{k}-1)$ with zero-order holder, then convert it with a adder.

$$
u(k)=u(k-1)+K \sum_{i=1}^{3} w_{i}(k) x_{i}(k)
$$

where, $w_{i}(\mathrm{k})$ is weight of $x i(\mathrm{k})$.

The supervised Hebb learning rule is adopted in this paper. The rule is:

$$
\Delta W_{i j}=\eta\left(d_{j}(k)-o_{j}(k)\right) o_{i}(k) o_{j}(k)
$$

where, $O_{i}$ is the active value of neuron $i, O_{j}$ is the active value of neuron $j, \Delta W_{i j}$ is the increment of the connection weight of neuron $i$ and neuron $j, d_{j}$ is expect output of the network object, and $\eta$ is learning efficiency.

Single neuron PID controller brings functions of self-adaptive and self-organization into effect by means of adjusting weights of quantities of state. In order to ensure the constringent controlled robustness of the learning strategy of single neuron adaptive control system, normalize the supervised Hebb learning rule: 


$$
\begin{aligned}
& u(k)=u(k-1)+K \sum_{i=1}^{3} w_{i}{ }^{\prime}(k) x_{i}(k) \\
& w_{i}{ }^{\prime}(k)=w_{i}(k) / \sum_{i=1}^{3}\left|w_{i}(k)\right| \\
& w_{1}(k)=w_{1}(k-1)+\eta_{I} z(k) u(k) x_{1}(k) \\
& \left.w_{2}(k)=w_{2}(k-1)+\eta_{p} z(k) u(k) x_{2}(k)\right\} \\
& w_{3}(k)=w_{3}(k-1)+\eta_{D} z(k) u(k) x_{3}(k)
\end{aligned}
$$

where, $\eta_{I}, \eta_{P}$ and $\eta_{P}$ are respectively the learning efficiency of integration, proportion and derivation [8].

According to the writing habit of S-Function and the functions provide by Matlab, single neuron control algorithm with S-Function is written like:

function [sys, $x 0]=\operatorname{sfn}(t, x, u, f l a g, n i, n p, n d, k)$

where, $t, x$ and $u$ are respectively time, state and input signal, and flag is flag bit. The initial values of neuron weight $W_{i}(i=1,2,3)$ equal respectively $0.3,0.3$ and 0.3 for this controller. $n i, n p, n d$ stand respectively for the learning efficiencies of integration, derivation and proportion. $k$ stands for the amplification of the neuron.

\section{Simulation experiment and results}

The process of the simulation. The selection of simulation algorithm: different solvation algorithms can be selected from the dialog box of Solver option. Generally, variable-step ode45 algorithm should be selected in the simulation of continuous system, while variable-step ode15 algorithm in rigidity problem and fixed-step discrete algorithm generally in discrete system. Because this simulation experiment is a continuous system, variable-step ode 45 algorithm is select.

The transfer function of the actuator in this experiment, mass flow meter, is considered as a second-order system in this simulation, for it is of some hysteresis nature and the actuator itself has certain PI compensation.

The assigned transfer function in second-order system is

$$
G(s)=\frac{2}{s^{2}+3 s+2} .
$$

the system simulation using single neuron control and common PID control are respectively processed as followed.

\section{(1) Single neuron module simulation}

Process: the inputs are transformed into three inputs of single neuron controller by state-converting, and then single neuron control module adjusts the coefficients of proportion, integration and derivation by calling S-Function. The block diagram is shown as Fig. 3. 


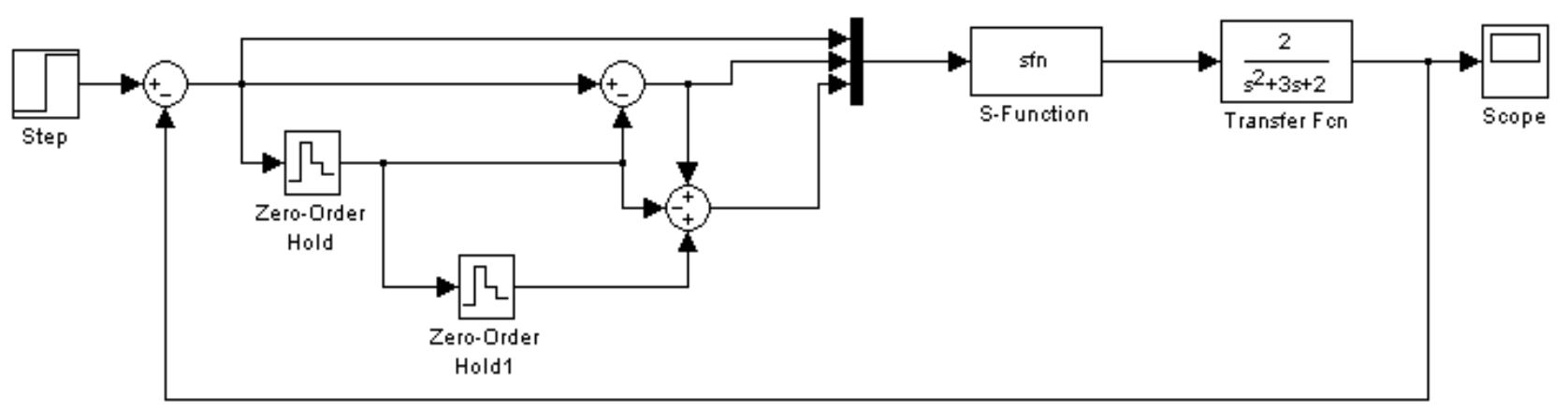

Fig. 3. Simulation structural design of a single neuron adaptive PID controller

Parameters setting: the initial learning efficiency $n o=[0.3,0.3,0.3$, the learning efficiency of integration $n p=680$, the learning efficiency of proportion $n i=65$, the learning efficiency of derivation $n d=100$, the proportional coefficient $k=20$. The waveform of the simulation result is shown as Fig. 4 . The overshoot is 0 , and the regulation time is $0.5 \mathrm{~s}$.

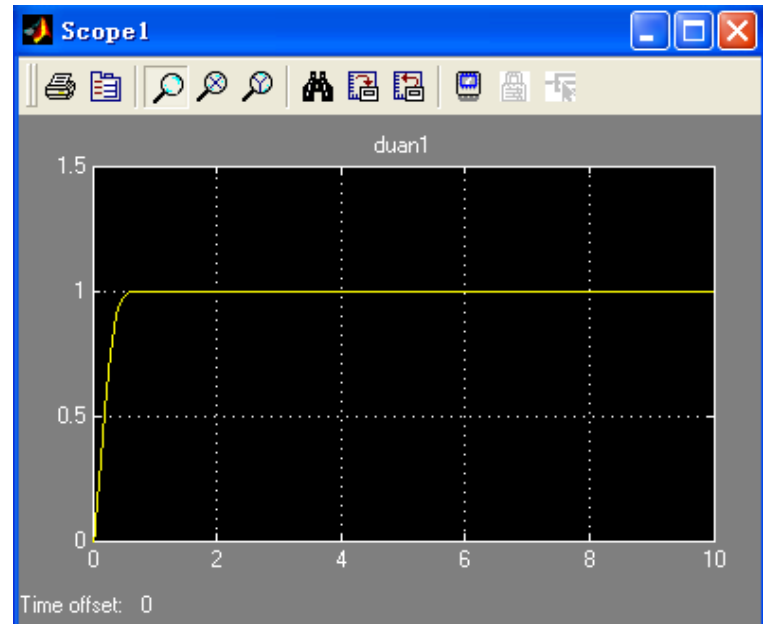

Fig. 4. Output waveform of a single neuron controller

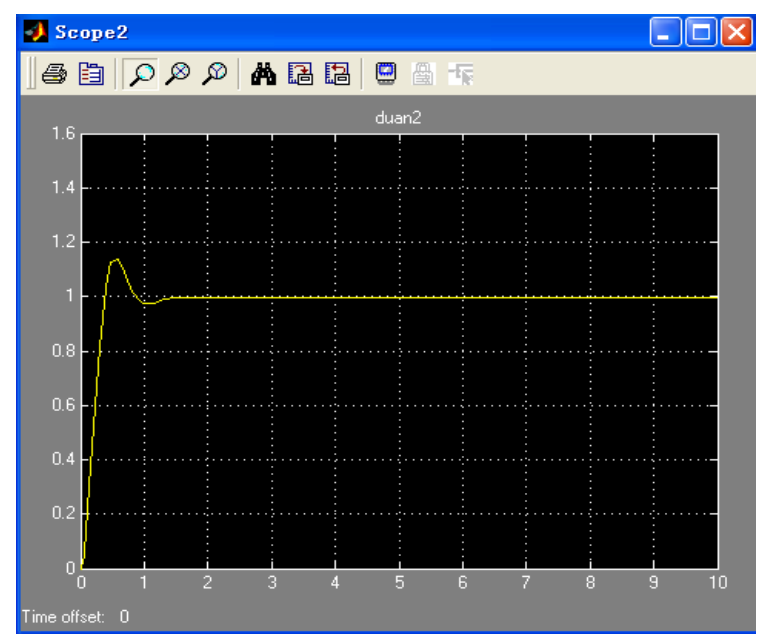

Fig. 6. Output waveform of conventional PID

\section{(2) Common PID control simulation}

Use PID controller module in Matlab. The block diagram is shown as Fig. 5.

Control parameter setting: when the parameters of PID are assigned respectively $\mathrm{P}=10, \mathrm{I}=10$ and $\mathrm{D}=10$, the waveform shown in Fig. 6 is more satisfactory. The overshoot is over $6 \%$, and the regulation time is more than $1.8 \mathrm{~s}$.

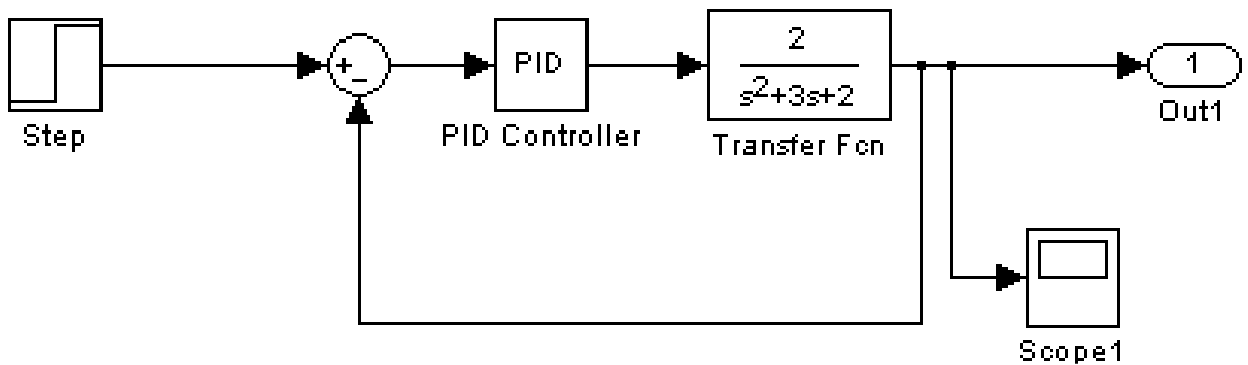

Fig. 5. Conventional PID controller block diagram

Analysis of simulation results. In real PEM control system, the differential value between the set-point and the feedback is regarded as step-function signal of simulation. Take monitoring the intensities of emission lines of reactive gas as an example to analyze the simulation results. It is true that the higher the spectrum intensity, the larger the particle density and the higher flow rate of the 
reactive gas. As shown in Fig. 4 and Fig. 6, when the waveform does not reach the equilibrium position, the sputtering state is in metal mode in which the film obtained is not chemical compound though the sputtering rate is high. The controller will output error signals to adjust the mass flow meter to increase the inlet flow rate of reactive gas until the waveform comes to the equilibrium state where the consumption is equal to the inlet of the reactive gas and the reaction goes steady. The overshot is over 6\%(Fig. 6), which illustrates that probably the reactive gas flow is excessive so it may lead to the target poisoning and reactive mode sputtering.

PEM control system using monomeric self-adapting PID algorithm with the less overshot and shorter regulation time can avoid target poisoning and responds faster than using non- self-adapting PID control.

\section{Conclusions}

In this paper, an automatic control system of quickly adjusting the gas mass flow according to the reactive state and single neuron self-adaptive control algorithm for PEM control system applied to the reactive coating machine is designed. Matlab is used to simulate the algorithm. Compared with the overshot $6 \%$ obtained by the non-adaptive PID algorithm, the lower result 0 by the single neuron self-adaptive PID algorithm shows that the target poisoning can be avoided and the reaction is in chemical mode. The comparison of the regulating time also shows that the PEM system using single neuron self-adaptive control algorithm responses faster than non-adaptive PID algorithm.

\section{References}

[1] H.D. Yang, C. Wu, H.B. Li, Y.H. Mai, et al., Online monitoring of VHF plasma optical emission spectra, Acta Physica Sinica, 52(2003) 2325-2327.

[2] S. Inoue, F. Okada, K. Koterazawa, CrN films deposited by r.f reactive sputtering using a plasma emission monitoring control, Vacuum, 66(2002) 227-231.

[3] S.K. Wu, Y.S. Chen, J.Z. Chen, Composition control of r.f.-sputtered Ti50Ni40Cu10 thin films using optical emission spectroscopy, Thin Solid Films, 365(2000) 61-66.

[4] E.M. McComb, H.D. Gesser, Analysis of trace metals in water by in-situ sample pre-concentration combined with wavelength dispersive X-ray fluorescence spectroscopy and inductively coupled plasma-optical emission spectroscopy, Talanta, 49(1999) 869-879.

[5] J.A. Morales, E.H. van Veen, M.T.C. de Loos-Vollebregt, Practical implementation of survey analysis in inductively coupled plasma optical emission spectrometry, Spectrochimica Acta, Part B, 53(1998) 683-697.

[6] Z.M. Wu, J. Sun, Q.S. Lei, Z.B. Ying, et al., Analysis on pressure dependence of microcrystalline silicon by optical emission spectroscopy, Physica E, 33(2006)125-129.

[7] X.Q. Zhu, Process monitoring, control technology and application, China Metallurgical Industry Press, Beijing, 2002, pp.156-170. 